\title{
Seroepidemiological survey for canine angiostrongylosis in dogs from Germany and the UK using combined detection of Angiostrongylus vasorum antigen and specific antibodies
}

\author{
M. SCHNYDER ${ }^{1}$, R. SCHAPER ${ }^{2}$, G. BILBROUGH ${ }^{3}$, E. R. MORGAN ${ }^{4}$ and P. DEPLAZES ${ }^{1}$ \\ ${ }^{1}$ Vetsuisse Faculty, Institute of Parasitology, University of Zurich, Winterthurerstrasse 266a, 8057 Zürich, Switzerland \\ ${ }^{2}$ Bayer Animal Health, Leverkusen, Germany \\ ${ }^{3}$ IDEXX Europe BV, Hoofddorp, the Netherlands \\ ${ }^{4}$ School of Veterinary Science, University of Bristol, Langford, BS40 5DU, UK
}

(Received 6 February 2013; revised 10 May and 6 Fune 2013; accepted 6 Fune 2013)

S U M MARY

Dogs infected with Angiostrongylus vasorum, a potentially lethal parasite parasitizing the heart and pulmonary arteries, may present severe respiratory, haematological and neurological signs. In this first large-scale seroepidemiological survey, 4003 sera originating from Germany and 4030 from the UK were tested by an ELISA for the detection of circulating antigen of $A$. vasorum, and by a separate ELISA detecting specific antibodies. In Germany, where mainly western federal states were sampled, $0 \cdot 3 \%(n=13$, CI: $0 \cdot 2-0 \cdot 6 \%)$ of dogs were positive in both ELISAs, whereas in total $0 \cdot 5 \%(n=20$, CI: $0 \cdot 3-0 \cdot 8 \%)$ were antigen-positive and $2 \cdot 25 \%(n=90, \mathrm{CI}: 1 \cdot 8-2 \cdot 8 \%)$ were positive for specific antibodies. Regions with antigen- and antibody-positive animals were overlapping. In the UK, where mainly the south of the country was sampled, $0 \cdot 97 \%(n=39$, CI: $0 \cdot 7-1 \cdot 3 \%)$ of dogs were antigen- and antibody positive. In total, $1 \cdot 32 \%(n=53, \mathrm{CI}: 1 \cdot 0-1 \cdot 7 \%)$ were antigen-positive, and $3 \cdot 2 \%(n=129$, CI: $2 \cdot 7-3 \cdot 8 \%)$ were positive for specific antibodies, again in overlapping regions. These results confirm the occurrence of $A$. vasorum in a random dog population originating from large parts of the countries investigated. The use of the tests alone or in combination was considered as a function of their sensitivities and specificities, in order to guide efficient clinical and epidemiological application.

Key words: Angiostrongylus vasorum, dog, serological survey, antigen detection, antibody detection, UK, Germany.

\section{INTRODUCTION}

Angiostrongylus vasorum is a nematode parasite of the right heart and pulmonary arteries of dogs and foxes, and uses Gastropoda as intermediate hosts (Guilhon, 1963; Rosen et al. 1970). The parasite was first described in France in the 19th century (Serres, 1854), followed by reports of endemic foci in southwestern France (Cuillé and Darraspen, 1930), Ireland (Roche and Kelliher, 1968), Switzerland (Wolff et al. 1969), England (Jacobs and Prole, 1975) and Italy (Poli et al. 1984). During recent decades, A. vasorum has increasingly been reported in extended areas in central and western European countries and also in Canada (Conboy, 2004) in dogs and wild carnivores. In Germany, single case reports (Barutzki and Schaper, 2003; Staebler et al. 2005; Denk et al. 2009) as well as epidemiological studies (Taubert et al. 2008; Barutzki and Schaper, 2009) have been performed based on canine fecal analyses. Angiostrongylus vasorum was described mainly in south-western regions. In the $\mathrm{UK}$ the presence of $A$. vasorum was originally described to be restricted

\footnotetext{
* Corresponding author: Vetsuisse Faculty, Institute of Parasitology, University of Zurich, Winterthurerstrasse 266a, 8057 Zürich, Switzerland. E-mail: manuela. schnyder@uzh.ch
}

to southern parts of the country (southern England, Wales) (Simpson and Neal, 1982; Chapman et al. 2004). Angiostrongylus vasorum has also long been present in the Republic of Ireland (Roche and Kelliher, 1968; Gallagher et al. 2012). A postmortem survey of foxes, the main wild reservoir of the parasite, suggested northward spread within the UK (Morgan et al. 2008), as confirmed by recent reports of cases in dogs from northern parts of the UK, including Scotland (Hayes and Rowlands, 2004; Helm et al. 2009; Yamakawa et al. 2009).

Infected dogs classically present with respiratory symptoms but clinical signs may be highly variable (Koch and Willesen, 2009; Schnyder et al. 2010), making the diagnosis of $A$. vasorum infections particularly challenging. Due to the often chronic and subtle course of the infection, owners often become aware only when severe symptoms such as coagulopathies or neurological disorders (Wessmann et al. 2006; Gredal et al. 2011) have established, with frequently fatal outcome (Staebler et al. 2005; Traversa et al. 2008; Denk et al. 2009; Yamakawa et al. 2009). Therefore, knowledge of the epidemiological situation concerning the presence of $A$. vasorum is fundamental for both animal owners and practising veterinarians. In known endemic areas, prevention of clinical angiostrongylosis is 
recommended through monthly use of macrocyclic lactones (Schnyder et al. 2009) or routine screening of dogs for $A$. vasorum infection (Verzberger-Epshtein et al. 2008; Schnyder et al. 2011).

Currently, diagnosis of $A$. vasorum infections is mainly based on the detection of first-stage larvae (L1) in fecal samples by larval migration methods such as the Baermann technique (Deplazes et al. 2012). Patency is described to start after 38-57 days post infection (dpi) (Bolt et al. 1994; Schnyder et al. 2010), when damage to the lung parenchyma is already present (Guilhon and Cens, 1969; Neff, 1971). Intermittent larval excretion has been described (Oliveira-Junior et al. 2006) and, therefore, repeated testing of fecal samples is suggested, in order to increase diagnostic sensitivity. Alternative diagnostic tools such as molecular methods (Jefferies et al. 2009; Al-Sabi et al. 2010) or highly specific serological tests have been developed (Schnyder et al. 2011; Schucan et al. 2012). ELISAs (Enzyme-linked immunosorbent assays) represent a valid and affordable tool for diagnosis in both individual and population studies, and have been very recently validated for $A$. vasorum infection in a field study (Guardone et al. 2013).

The objective of this research was to perform a sero-epidemiological survey of circulating antigens of $A$. vasorum and of specific antibodies against A. vasorum in sera of dogs originating from Germany and the UK. In addition, independent estimates of test sensitivities and specificities were used in the context of observed prevalence in this study to consider how each test might be efficiently used alone or in combination to support clinical and epidemiological investigations.

\section{MATERIALS AND METHODS}

Sera of 4003 dogs from Germany and 4030 dogs from the $\mathrm{UK}$ were submitted to private veterinary diagnostic laboratories (Vet Med Labor GmbH, Idexx Laboratories, Ludwigsburg in Germany, and Idexx Laboratories Ltd, Southwater and Idexx Laboratories Ltd, Wetherby in the UK) for various medical reasons. Samples from Germany originated from western federal states and northern Bavaria, while samples from the UK were obtained from widespread areas of England and Wales, with a concentration in the south-east.

All samples were stored at $-20^{\circ} \mathrm{C}$ before and after sending them to the Institute of Parasitology in Zurich, where they were analysed for the presence of circulating $A$. vasorum antigens in a sandwichELISA using mono- and polyclonal antibodies (sensitivity $95 \cdot 7 \%$, specificity $94 \cdot 0 \%$ ), as previously described (Schnyder et al. 2011). Additionally, an ELISA detecting specific antibodies using excretory/ secretory (E/S) antigens of adult stages (sensitivity $85 \cdot 7 \%$, specificity $98 \cdot 8 \%$ ) was used (Schucan et al.
2012). Test thresholds were regionally determined with 300 randomly selected samples based on the mean value of optical density $\left(\mathrm{A}_{405 \mathrm{~nm}}\right)$ plus 3 (antigen detection) or 4 (antibody detection) standard deviations (S.D.).

The collected data were analysed by a geographic information system (GIS) using the program RegioGraph 10 (GfK GeoMarketing, Bruchsal, Germany) to visualize the regional distribution of collected and analysed serum samples and $A$. vasorum antigen and/or antibody positive samples. Based on the four digit (UK) and five-digit postcodes (Germany) as points of reference, the locations of positive samples were displayed on maps with administrative and postcode boundaries. Due to privacy protection, no further data concerning the animal or the animal owner were available. Excel 2007 for Windows (Microsoft Corporation, Redmond, USA) was used to calculate the prevalence values, the confidence interval (CI) of the sensitivities and the specificities and for the calculation of means and S.D.

The sensitivity (SE) and specificity (SP) of combined tests were calculated from those of individual tests (denoted by suffix $a$ or $b$ ) using the method described by Weinstein et al. (2005). Thus, test results can be combined using the OR rule, i.e. the combined test is positive if either individual test returns a positive result, and negative only if both individual tests are negative. Alternatively, using the AND rule, the combined test is positive only if both individual tests are positive, and negative if either individual test is negative. Using the $\mathrm{OR}$ rule, combined $\mathrm{SE}=\mathrm{SE}_{a}+\mathrm{SE}_{b}-\left(\mathrm{SE}_{a} \times \mathrm{SE}_{b}\right)$, and combined $\mathrm{SP}=\left(\mathrm{SP}_{a} \times \mathrm{SP}_{b}\right)$. Using the $\mathrm{AND}$ rule, combined $\mathrm{SE}=\left(\mathrm{SE}_{a} \times \mathrm{SE}_{b}\right)$, and combined $\mathrm{SP}=$ $\mathrm{SP}_{a}+\mathrm{SP}_{b}-\left(\mathrm{SP}_{a} \times \mathrm{SP}_{b}\right)$. Positive predictive value (PPV), which depends also on the population prevalence of infection (prev), was calculated as: $\mathrm{PPV}=$ $(\mathrm{SE} \times$ prev $) /(\mathrm{SE} \times$ prev $)+(1-\mathrm{SP})(1-$ prev $))$.

\section{RESULTS}

The location and seropositivity of all tested samples in Germany, where mainly western federal states were sampled, is shown in Fig. 1. A total of $0 \cdot 3 \%$ ( $n=13$, confidence intervals, CI: $0 \cdot 2-0 \cdot 6 \%$ ) of the animals were positive in both ELISAs, while $0 \cdot 5 \%$ $(n=20$, CI: $0 \cdot 3-0 \cdot 8 \%$ ) of the tested dogs were antigen-positive and $2 \cdot 25 \%(n=90, \mathrm{CI}: 1 \cdot 8-2 \cdot 8 \%)$ were positive for specific antibodies. Regions with antigen- and antibody-positive animals were overlapping and distributed through the whole western sampling area of the country.

The location and results of serological analysis of sera from the UK, where mainly the south of the country was sampled, are shown in Fig. 2A (UK) and 2B (southern England and Wales, with enlargement of the area around London for details). A total of 


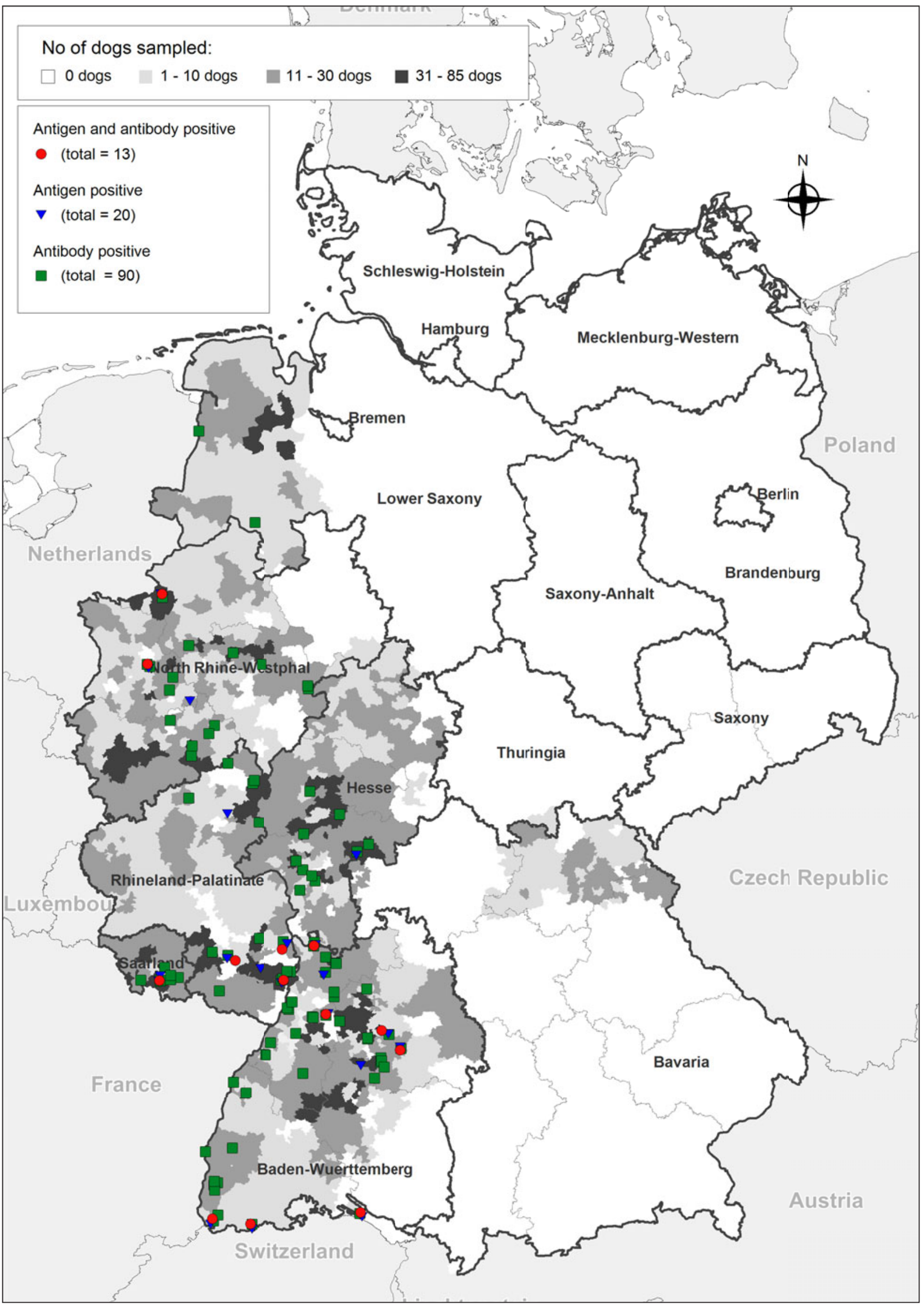

Fig. 1. Serological results of 4003 sera collected from western federal states in Germany, tested for the presence of circulating Angiostrongylus vasorum antigens (Schnyder et al. 2011) and for the presence of $A$. vasorum specific antibodies (Schucan et al. 2012). Test thresholds were regionally determined with 300 randomly selected samples based on the mean value of optical density (A405 nm) plus 3 (antigen detection) or 4 (antibody detection) standard deviations. 
Table 1. Sensitivity and specificity of the two serological tests for detection of Angiostrongylus vasorum, alone and in combination

\begin{tabular}{lllll}
\hline \hline & $\begin{array}{l}\text { Ab- } \\
\text { ELISA }\end{array}$ & $\begin{array}{l}\text { Ag- } \\
\text { ELISA }\end{array}$ & $\begin{array}{l}\text { Ab OR } \\
\mathrm{Ag}\end{array}$ & $\begin{array}{l}\text { Ab AND } \\
\mathrm{Ag}\end{array}$ \\
\hline Sensitivity & 0.857 & 0.957 & 0.994 & 0.820 \\
Specificity & 0.988 & 0.940 & 0.929 & 0.999 \\
\hline \hline
\end{tabular}

Ab-ELISA = antibody detection, representing parasite exposure; Ag-ELISA = antigen detection, representing an actual infection; $\mathrm{Ab}$ OR Ag is positive if either individual test is positive, and negative if both are negative; $\mathrm{Ab}$ AND $\mathrm{Ag}$ is positive only if both individual tests are positive, and negative if either is negative. Combining tests by the ' $O R$ ' rule enhances sensitivity at the cost of specificity, while the 'AND' rule enhances specificity but at the cost of sensitivity.

$0 \cdot 97 \% \quad(n=39, \mathrm{CI}: 0 \cdot 7-1 \cdot 3 \%)$ of the dogs were antigen- and antibody positive. Overall, $1 \cdot 32 \%$ ( $n=53$, CI: $1 \cdot 0-1 \cdot 7 \%)$ of the animals were antigenpositive, and $3 \cdot 2 \%(n=129, \mathrm{CI}: 2 \cdot 7-3 \cdot 8 \%)$ were positive for specific antibodies, again in overlapping regions.

The effect of combining antigen and antibody detection tests is summarized in Table 1, where it has to be considered that antigen detection represents an actual infection, while specific $\mathrm{Ab}$ detection represents a parasite exposure. When using the OR rule, since the combined test is deemed positive if either individual test yields a positive result, overall sensitivity is enhanced, but at the cost of decreased overall specificity. Conversely, using the AND rule, both tests must be positive to return a combined positive result and, as this is harder to achieve, specificity is higher than that of either individual test, but sensitivity is lower. Variation in the PPV of different tests and combinations with changing population prevalence of infection with $A$. vasorum is shown in Fig. 3.

\section{DISCUSSION}

Results confirmed the endemic presence of $A$. vasorum in Germany as well as in the $\mathrm{UK}$, and additionally cases outside the known endemic foci were detected. The use of ELISA techniques allowed for the first time a large sero-epidemiological survey in these two countries. Thus, this is the largest survey of this emerging parasite so far conducted, and the first to report rates of infection in dogs in multiple endemic countries using the same tests. Moreover, it introduces the concept of using both antigen and antibody detection ELISAs for epidemiological and clinical diagnosis, offering great potential for future research and application. Sensitivity and specificity of both tests had been previously assessed (Schnyder et al. 2011; Schucan et al. 2012) and both tests had been validated in a field study in which serological results were compared with results of Baermann analyses (Guardone et al. 2013). ELISAs were shown to be robust, giving coherent results, i.e. with haemolytic or lipaemic sera or when thawed and frozen several times. Baermann techniques with isolation of L1 are routinely applied for diagnosis of lungworms, with limitations concerning sensitivity (as a consequence of irregular larval shedding, the difficulties of collecting multiple fecal samples and of guaranteeing proper storage of the samples before analysis) and also specificity, if not performed by experienced technicians. ELISAs were shown to positively confirm samples from Baermann-positive dogs, and additionally detected non-patent infections (Guardone et al. 2013), and are therefore highly sensitive methods. However, interpretation of the two ELISA tests differs: while specific antibody detection is useful for the determination of parasite exposure, antigen detection represents an actual infection. This explains the higher number of positive dogs using antibody detection compared with antigen detection (90/20 and 129/53 for Germany and the UK, respectively). Implications of positive results with each or both individual tests go beyond those of manipulating overall sensitivity and specificity. Thus, dogs positive for both tests, in which circulating antigens as well as specific antibodies against $A$. vasorum are detected, can be assumed to harbour an active $A$. vasorum infection inducing an immunological reaction with production of antibodies. Dogs in which only antibodies are detected may have acquired $A$. vasorum recently, since antibodies can already be detected $21 \mathrm{dpi}$, while antigen is first detected $35 \mathrm{dpi}$ (Schnyder et al. 2011; Schucan et al. 2012). Alternatively, antibody-positive dogs may be parasite-free, i.e. after efficacious anthelmintic treatment or natural clearance of infection: dogs become seronegative for antigen detection within 16-34 days after single treatment with imidacloprid/moxidectin (Advocate ${ }^{\circledR}$ spot on) (Schnyder et al. 2011), while antibodies persist up to 63 days after treatment (Schucan et al. 2012). Furthermore, cross-reactions and false positive results cannot be completely excluded, although the indicated specificities, based on sera of dogs infected with other common (Toxocara canis, Ancylostoma caninum, Leishmania sp.) and uncommon (Dirofilaria immitis, Dirofilaria repens, Crenosoma vulpis, Eucoleus aerophilus) parasitic infections, are high (Schnyder et al. 2011; Schucan et al. 2012).

While the performance of the tests, alone or in combination, is predictable from their independently estimated sensitivities and specificities, their PPV depends also on population prevalence of infection. This should influence the way in which the tests are used in practice (Pepe and Thompson, 2000). Thus, for instance, in a situation of surveillance for the early appearance of angiostrongylosis in an area of low 


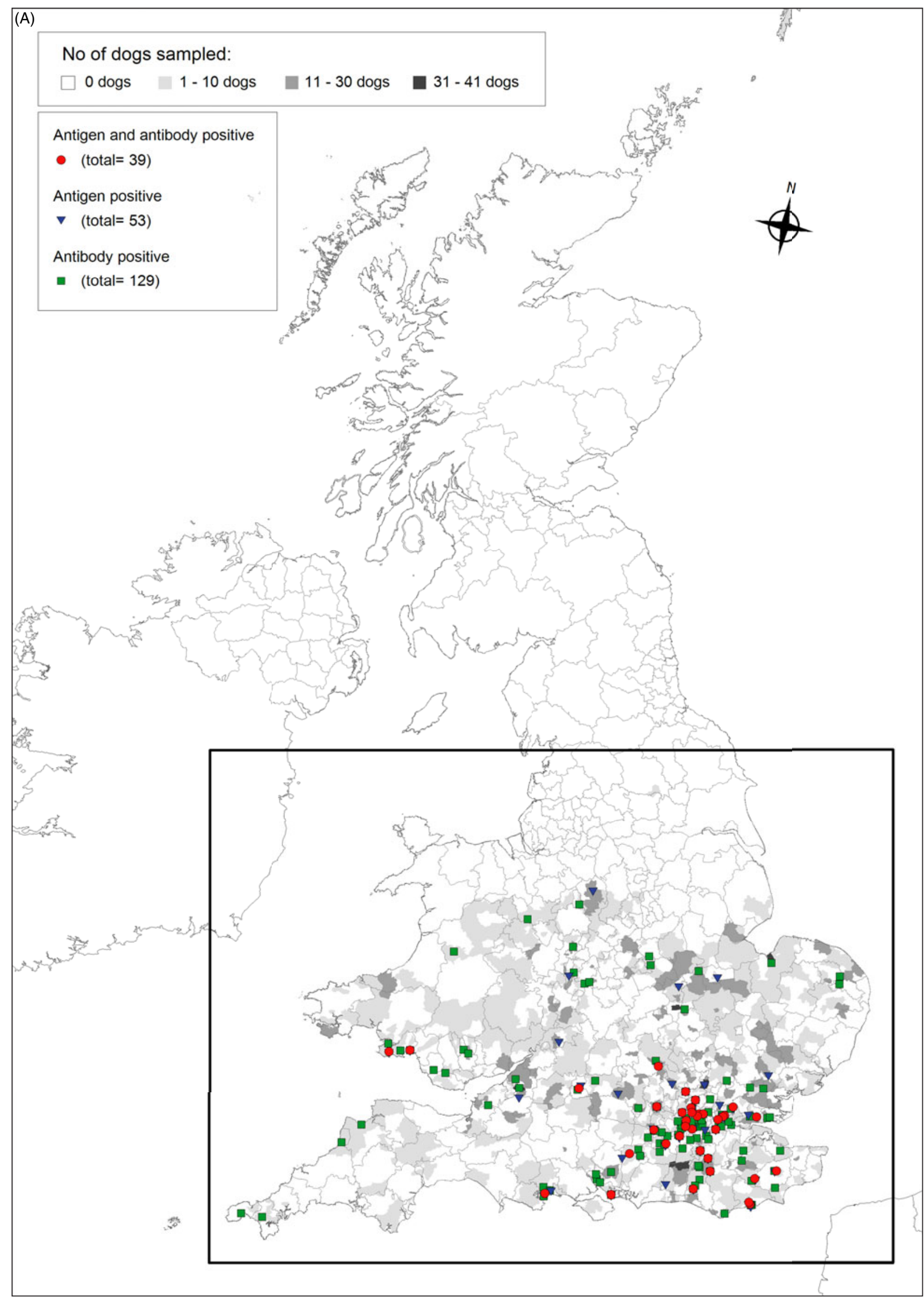

Fig. 2. See the following page for legend. 
(B)

No of dogs sampled:
$\square 0$ dogs $\square 1$ - 10 dogs $\square 11$ - 30 dogs $\square 31-41$ dogs
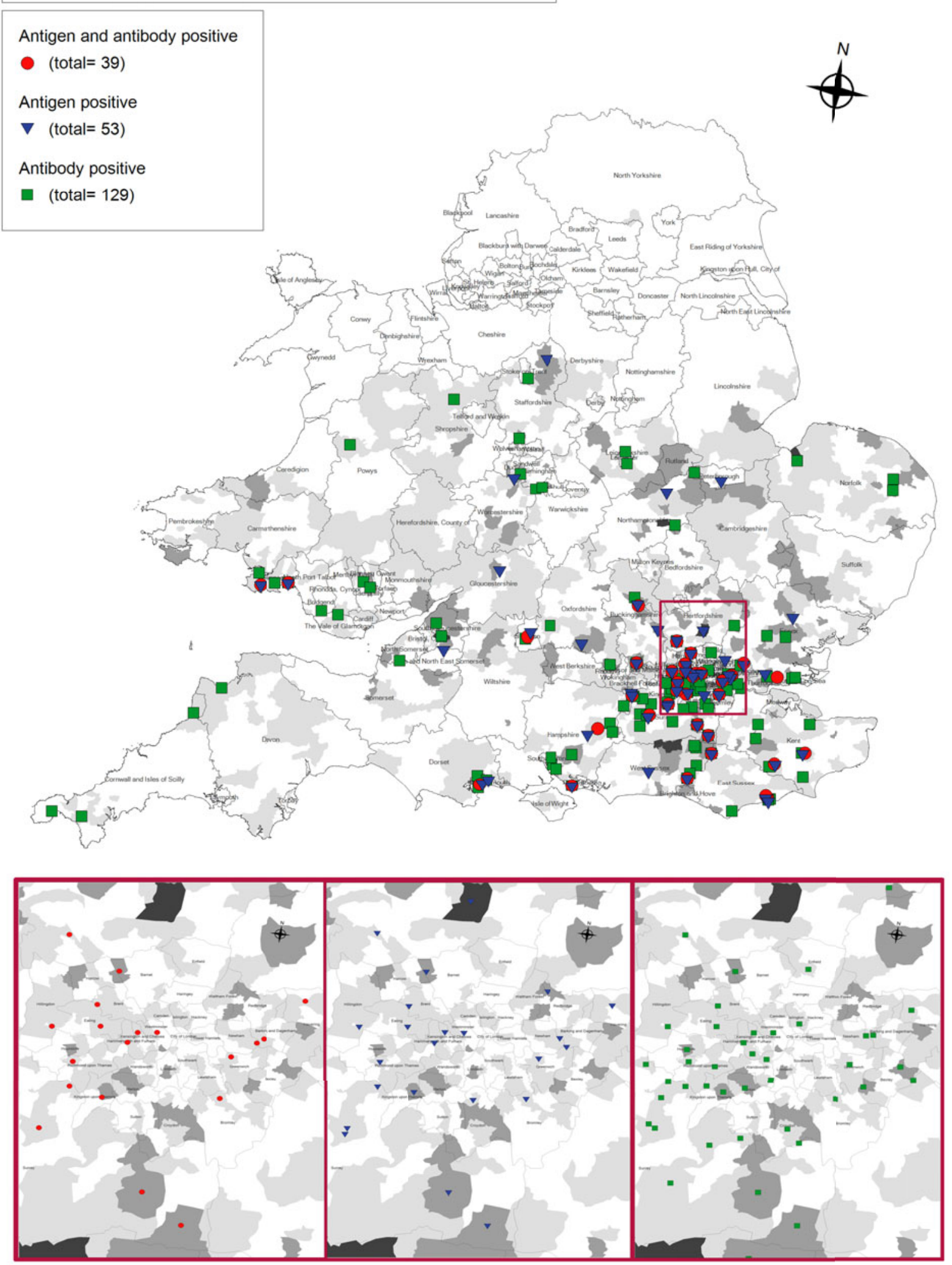

Fig. 2. (A) Overview of the UK and the results of the seroepidemiological survey for canine angiostrongylosis;

(B) Serological results of 4030 sera collected from England and Wales. Results of dogs from London and surroundings are additionally represented separately because of overlapping symbols. For details of test performance see Fig. 1. 


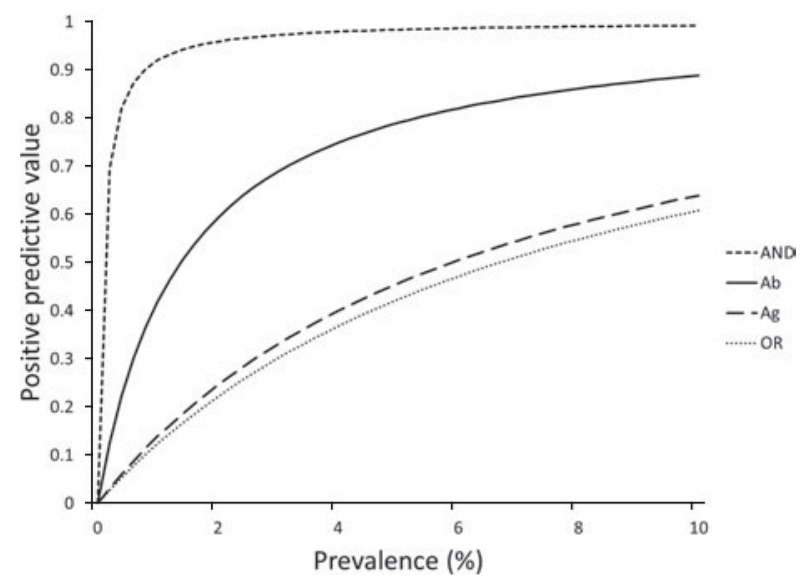

Fig. 3. Positive predictive value of the serological tests, i.e. the proportion of positive test results that indicate a truly positive sample. $\mathrm{Ab}=$ antibody-detection ELISA (continuous curve); $\mathrm{Ag}=$ antigen-detection ELISA (large dashed curve); $\mathrm{AND}=$ positive if both tests are positive ( short dashed curve), OR = positive if either test is positive (dotted curve). Ag detection and the combined antigen plus specific $\mathrm{Ab}$ detection represent actual infections, while specific $\mathrm{Ab}$ detection represents a parasite exposure. At low prevalence, tests with high specificity have a particularly strong advantage in terms of positive predictive value, in this case the combined AND test. Positive predictive value generally trades off against sensitivity (see Table 1).

expected prevalence, if both tests are used together and a positive result declared only if both individual tests are positive, the PPV is substantially increased and therefore the risk of false negatives decreased. By contrast, clinical diagnosis in an area of known high prevalence might efficiently use the more sensitive antigen detection test first, and perhaps confirm positives using the more specific antibody detection test. When assessing the cost of misclassification error, it should be noted that treatment of false positives is much less grave clinically than nontreatment of false negative cases, since these could succumb to severe and possibly fatal disease. The information provided in Table 1 and Fig. 3 should enable users to choose an appropriate strategy for their purpose.

In epidemiological surveys, the trade-off between sensitivity and specificity is further affected by sample size. Thus, decreased sensitivity (in favour of increased specificity, e.g. using the AND rule) is less likely to miss infection where it is really present if sample size is large. The data presented here will assist in the design and power analysis of such surveys. As a result, increased information and disease awareness will lead to more appropriate testing. The combined use of antigen and antibody detection ELISA offers great potential for early and sensitive diagnosis of these infections, which are otherwise potentially lethal, mainly due to coagulopathies and neurological problems in cases not subjected to timely diagnosis (Staebler et al. 2005; Traversa et al. 2008; Denk et al. 2009; Yamakawa et al. 2009). Accordingly, adequate anthelmintic treatments can be implemented. This is also essential to prevent pathological changes, which can be present in lungs early during infection (Neff, 1971; Schnyder et al. 2009; Dennler et al. 2011). Due to privacy protection, no further information concerning the tested animals was available, and no Baermann analysis of fecal samples for confirmation of serologically positive samples was possible. Besides, it was not possible to give feedback to veterinarians in charge of serologically positive animals to encourage their treatment.

The sero-prevalences obtained in this study among dogs sampled for various diseases cannot be directly compared with prevalence data obtained through testing fecal samples of dogs with the Baermann technique. Nevertheless, an evident overlapping of the areas in Germany with positive dogs is observed: in a recently published study, a prevalence of $7 \cdot 4 \%$ was detected in dogs originating from approximately the same areas, selected for cardiorespiratory signs, and tested with fecal samples collected over 3 days (Barutzki and Schaper, 2009). In another study with pre-selected dogs having clinical signs of potential lungworm infections, prevalence tested with fecal samples collected on a single day was $1 \cdot 2 \%$ (Taubert et al. 2008); these samples were collected from larger parts of Germany, with positives also originating mainly from western and southern areas of the country. Sera in the present study were submitted for miscellaneous but unknown medical reasons, including cardiorespiratory signs; due to their high number the dataset is considered representative of an average canine population.

Previous population studies in dogs based on fecal analyses in the UK, as previously reviewed (Morgan et al. 2010), were restricted to areas of London in stray/re-homed dogs (with a prevalence of 1.8\%) and Cornwall (Martin and Neal, 1992) in pre-selected dogs showing compatible clinical signs (prevalence: $4 \cdot 1 \%)$. A geographical summary of canine cases from 2009 (Yamakawa et al. 2009) showed that canine angiostrongylosis, once restricted to Wales and southern England, is expanding to northern England, with single case reports in that region complemented by a single case reported from Scotland (Helm et al. 2009). A questionnaire survey sent to 3950 small animal practices during 2009 confirmed that $A$. vasorum appears to be spreading beyond the traditional endemic foci in the UK (Ashley et al. 2012). This situation was anticipated by a study on 546 foxes culled between 2005 and 2006 (Morgan et al. 2008). The detected prevalence in the present study of up to $3 \cdot 2 \%$ (antibody detection) confirms the presence of $A$. vasorum in non-selected dogs from Wales and large parts of central and southern England. 
In conclusion, diagnostic testing with ELISAs enables detection of infection with $A$. vasorum shortly before or contemporaneously with patency, requires a single serum sample instead of repeated fecal samples, and has the potential for a rapid diagnostic test, particularly in the case of a large number of samples. The detection of circulating antigens and specific antibodies against $A$. vasorum by ELISA therefore represents a useful and reliable tool for population studies as shown for large areas of Germany and the UK. The combined use of antigen and antibody detection ELISA offers great potential for future research and application for epidemiological and clinical diagnosis. Increased knowledge concerning the epidemiological situation and therefore disease awareness of this potentially fatal parasite of dogs allows early diagnosis of the infection. This is essential to ensure adequate treatments in case of severe canine angiostrongylosis but also holds true for dogs at early stages of infection, therefore avoiding the onset of pathological changes, which are possible even in the absence of clinical signs. Furthermore, in known endemic areas prophylactic anthelmintic treatment or routine screening of dogs for $A$. vasorum infection are recommended. This is particularly important considering the confirmed establishment and potential expansion of $A$. vasorum in various European countries.

\section{ACKNOWLEDGEMENTS}

We are very grateful to Dr Iskanderali Ziadinov and Isabelle Tanner from the Institute of Parasitology in Zurich for their very valuable support and Carola Hafner and David Fox for the organization of the samples.

\section{FINANCIAL SUPPORT}

This work was supported by Bayer AG.

\section{REFERENCES}

Al-Sabi, M. N., Deplazes, P., Webster, P., Willesen, J. L., Davidson, R. K. and Kape1, C. M. (2010). PCR detection of Angiostrongylus vasorum in faecal samples of dogs and foxes. Parasitology Research 107, 135-140. doi: 10.1007/s00436-010-1847-5.

Ashley, L., Limon, G., Guitian, J., Hermosilla, C. and Fox, M. T. (2012). Angiostrongylus vasorum in the United Kingdom - a nationwide postal questionnaire survey. In 2nd Bayer Angiostrongylosis Forum Including Major Parasitic Lung Diseases of Dogs and Cats (ed. Schaper, R.), Parma, Italy, 22nd June 2012.

Barutzki, D. and Schaper, R. (2003). Endoparasites in dogs and cats in Germany 1999-2002. Parasitology Research 90(Suppl.), S148-S150. doi: 10.1007/s00436-003-0922-6.

Barutzki, D. and Schaper, R. (2009). Natural infections of Angiostrongylus vasorum and Crenosoma vulpis in dogs in Germany (2007-2009). Parasitology Research 105(Suppl.), S39-S48. doi: 10.1007/s00436-0091494-x.

Bolt, G., Monrad, J., Koch, J. and Jensen, A.L. (1994). Canine angiostrongylosis: a review. Veterinary Record 135, 447-452.

Chapman, P.S., Boag, A. K., Guitian, J. and Boswood, A. (2004). Angiostrongylus vasorum infection in 23 dogs (1999-2002). Fournal of Small Animal Practice 45, 435-440.
Conboy, G. (2004). Natural infections of Crenosoma vulpis and Angiostrongylus vasorum in dogs in Atlantic Canada and their treatment with milbemycin oxime. Veterinary Record 155, 16-18.

Cuillé, J. and Darraspen, E. (1930). De la Strongylose cardio-pulmonaire du chien. Revue Générale de Médecine Vétérinaire 466, 625-639, 694-710.

Denk, D., Matiasek, K., Just, F. T., Hermanns, W., Baiker, K., Herbach, N., Steinberg, T. and Fischer, A. (2009). Disseminated angiostrongylosis with fatal cerebral haemorrhages in two dogs in Germany: a clinical case study. Veterinary Parasitology 160, 100-108. doi: 10.1016/ j.vetpar.2008.10.077.

Dennler, M., Makara, M., Kranjc, A., Schnyder, M., Ossent, P., Deplazes, P., Ohlerth, S. and Glaus, T. M. (2011). Thoracic computed tomography findings in dogs experimentally infected with Angiostrongylus vasorum. Veterinary Radiology and Ultrasound 52, 289-294. doi: 10.1111/ j.1740-8261.2010.01776.x.

Deplazes, P., Eckert, J., v. Samson-Himmelstjerna, G. and Zahner, H. (2012). Lehrbuch der Parasitologie für die Tiermedizin. Enke Verlag, Stuttgart, Germany.

Gallagher, B., Brennan, S. F., Zarelli, M. and Mooney, C. T. (2012). Geographical, clinical, clinicopathological and radiographic features of canine angiostrongylosis in Irish dogs: a retrospective study. Irish Veterinary fournal 65, 5. doi: 10.1186/2046-0481-65-5.

Gredal, H., Willesen, J.L., Jensen, H. E., Nielsen, O. L., Kristensen, A. T., Koch, J., Kirk, R. K., Pors, S. E., Skerritt, G. C. and Berendt, M. (2011). Acute neurological signs as the predominant clinical manifestation in four dogs with Angiostrongylus vasorum infections in Denmark. Acta Veterinaria Scandinavica 53, 43. doi: 10.1186/1751-014753-43.

Guardone, L., Schnyder, M., Macchioni, F., Deplazes, P. and Magi, M. (2013). Serological detection of circulating Angiostrongylus vasorum antigen and specific antibodies in dogs from central and northern Italy. Veterinary Parasitology 192, 192-198. doi: org/10.1016/ j.vetpar.2012.10.016.

Guilhon, J. (1963). Recherches sur le cycle évolutif du Strongle des vaisseaux du chien. Bulletin de l'Académie Vétérinaire 36, 431-442.

Guilhon, J. and Cens, B. (1969). Migrations and evolution of Angiostrongylus vasorum (Baillet, 1866) in dogs. Comptes Rendus Hebdomadaires des Seances de l'Academie des Sciences - D: Sciences Naturelles 269, 2377-2380. (In French)

Hayes, G. and Rowlands, M. (2004). Angiostrongylus infection in a dog in north-west England. Veterinary Record 154, 639.

Helm, J., Gilleard, J. S., Jackson, M., Redman, E. and Bell, R. (2009). A case of canine Angiostrongylus vasorum in Scotland confirmed by PCR and sequence analysis. Fournal of Small Animal Practice 50, 255-259. doi: 10.1111/j.1748-5827.2009.00741.x.

Jacobs, D. E. and Prole, J. H. (1975). Angiostrongylus vasorum and other nematodes in British greyhounds. Veterinary Record 96, 180.

Jefferies, R., Morgan, E. R. and Shaw, S. E. (2009). A SYBR green realtime PCR assay for the detection of the nematode Angiostrongylus vasorum in definitive and intermediate hosts. Veterinary Parasitology 166, 112-118. doi: 10.1016/j.vetpar.2009.07.042.

Koch, J. and Willesen, J. L. (2009). Canine pulmonary angiostrongylosis: an update. Veterinary fournal 179, 348-359. doi: 10.1016/j.tvj1.2007.11.014. Martin, M. and Neal, C. (1992). Distribution of angiostrongylosis in Cornwall. Fournal of Small Animal Practice 33, 327-336.

Morgan, E. R., Tomlinson, A., Hunter, S., Nichols, T., Roberts, E., Fox, M. T. and Taylor, M.A. (2008). Angiostrongylus vasorum and Eucoleus aerophilus in foxes (Vulpes vulpes) in Great Britain. Veterinary Parasitology 154, 48-57. doi: 10.1016/j.vetpar.2008.02.030.

Morgan, E. R., Jefferies, R., van Otterdijk, L., McEniry, R.B., Allen, F., Bakewell, M. and Shaw, S. E. (2010). Angiostrongylus vasorum infection in dogs: presentation and risk factors. Veterinary Parasitology $\mathbf{1 7 3}$, 255-261. doi: 10.1016/j.vetpar.2010.06.037.

Neff, H. (1971). Experimentelle Infektionen von Hunden mit Angiostrongylus vasorum (Nematoda). Dissertation. Universität Zürich, Zürich. (In German)

Oliveira-Junior, S. D., Barcante, J. M., Barcante, T. A., Dias, S. R. and Lima, W. S. (2006). Larval output of infected and re-infected dogs with Angiostrongylus vasorum (Baillet, 1866) Kamensky, 1905. Veterinary Parasitology 141, 101-106. doi: 10.1016/j.vetpar.2006.05.003.

Pepe, M.S. and Thompson, M. L. (2000). Combining diagnostic test results to increase accuracy. Biostatistics 1, 123-140. doi: 10.1093/ biostatistics/1.2.123.

Poli, A., Arispici, M., Marconcini, A., Mancianti, F. and de Monte, D. (1984). Angiostrongylus vasorum (Baillet, 1866) in red foxes (Vulpes vulpes L.) in Italy. Fournal of Wildlife Diseases 20, 345-346.

Roche, M. M. and Kelliher, D. J. (1968). Angiostrongylus vasorum infestation in the dog: a case report. Irish Veterinary fournal 22, 108-113. 
Rosen, L., Ash, L.R. and Wallace, G. D. (1970). Life history of the canine lungworm Angiostrongylus vasorum (Baillet). American fournal of Veterinary Research 31, 131-143.

Schnyder, M., Fahrion, A., Ossent, P., Kohler, L., Webster, P., Heine, J. and Deplazes, P. (2009). Larvicidal effect of imidacloprid/ moxidectin spot-on solution in dogs experimentally inoculated with Angiostrongylus vasorum. Veterinary Parasitology 166, 326-332. doi: 10.1016/j.vetpar.2009.09.004

Schnyder, M., Fahrion, A., Riond, B., Ossent, P., Webster, P., Kranjc, A., Glaus, T. and Deplazes, P. (2010). Clinical, laboratory and pathological findings in dogs experimentally infected with Angiostrongylus vasorum. Parasitology Research 107, 1471-1480. doi: 10.1007/s00436-0102021-9.

Schnyder, M., Tanner, I., Webster, P., Barutzki, D. and Deplazes, P. (2011). An ELISA for sensitive and specific detection of circulating antigen of Angiostrongylus vasorum in serum samples of naturally and experimentally infected dogs. Veterinary Parasitology 179, 152-158. doi: 10.1016/j.vetpar. 2011.01.054

Schucan, A., Schnyder, M., Tanner, I., Barutzki, D., Traversa, D. and Deplazes, P. (2012). Detection of specific antibodies in dogs infected with Angiostrongylus vasorum. Veterinary Parasitology 185, 216-224. doi: 10.1016/j.vetpar.2011.09.040.

Serres, E. (1854). Entozoaires trouvés dans l'oreille droite, le ventricule correspondant et l'artère pulmonaire d'un chien. Fournal des Vétérinaires du Midi 7, 70. (In French)

Simpson, V.R. and Neal, C. (1982). Angiostrongylus vasorum infection in dogs and slugs. Veterinary Record 111, 303-304.

Staebler, S., Ochs, H., Steffen, F., Naegeli, F., Borel, N., SieberRuckstuhl, N. and Deplazes, P. (2005). Autochthonous infections with
Angiostrongylus vasorum in dogs in Switzerland and Germany. Schweizer Archiv fur Tierheilkunde 147, 121-127. (In German)

Taubert, A., Pantchev, N., Vrhovec, M. G., Bauer, C. and Hermosilla, C. (2008). Lungworm infections (Angiostrongylus vasorum Crenosoma vulpis, Aelurostrongylus abstrusus) in dogs and cats in Germany and Denmark in 2003-2007. Veterinary Parasitology 159 , 175-180. doi: 10.1016/j.vetpar.2008.10.005.

Traversa, D., Torbidone, A., Malatesta, D. and Guglielmini, C. (2008). Occurrence of fatal canine Angiostrongylus vasorum infection in Italy. Veterinary Parasitology 152, 162-166. doi: 10.1016/j.vetpar. 2007.12.009.

Verzberger-Epshtein, I., Markham, R. J., Sheppard, J. A., Stryhn, H., Whitney, H. and Conboy, G. A. (2008). Serologic detection of Angiostrongylus vasorum infection in dogs. Veterinary Parasitology 151, 53-60. doi: 10.1016/j.vetpar.2007.09.028.

Weinstein, S., Obuchowski, N. A. and Lieber, M.L. (2005) Clinical evaluation of diagnostic tests. American fournal of Roentgenologists 184, 14-19.

Wessmann, A., Lu, D., Lamb, C. R., Smyth, B., Mantis, P., Chandler, K., Boag, A., Cherubini, G. B. and Cappello, R. (2006). Brain and spinal cord haemorrhages associated with Angiostrongylus vasorum infection in four dogs. Veterinary Record 158, 858-863.

Wolff, K., Eckert, J. and Leemann, W. (1969). Beitrag zur Angiostrongylose des Hundes. Dtsch Vet-med Ges, Zürich, Switzerland.

Yamakawa, Y., McGarry, J. W., Denk, D., Dukes-McEwan, J., Macdonald, N., Mas, A., McConnell, F., Tatton, B., Valentine, E. G., Wayne, J., Williams, J. M. and Hetzel, U. (2009). Emerging canine angiostrongylosis in northern England: five fatal cases. Veterinary Record 164, 149-152. 